\title{
Behavioural family therapy reduced disruptive behaviour in children at risk for developing conduct problems
}

\author{
Sanders MR, Markie-Dadds C, Tully LA, et al. The Triple P-Positive Parenting Program: a comparison of enhanced, \\ standard, and self-directed behavioral family intervention for parents of children with early onset conduct problems.J \\ Consult Clin Psychol 2000 Aug;68:624-40.
}

QUESTION: For families with 3 year old children at high risk for developing conduct problems, does a behavioural family intervention (BFI) (Triple-P Positive Parenting Program) in standard, enhanced, or self directed formats reduce disruptive child behaviour?

Design

Randomised (allocation concealed*), blinded (outcome assessors),* controlled trial with follow up at 1 year.

\section{Setting}

An outpatient clinic and community health and neighbourhood clinics in Queensland, Australia.

\section{Patients}

305 families with a 3 year old child ( $68 \%$ boys) were recruited through targeted advertisements. Inclusion criteria were maternal concern about the child's behaviour (increased score on maternal rating of the child's behaviour), no developmental disorder, the child or family were not receiving care for behaviour or psychological problems, and the family had $\geqslant 1$ family adversity factor. Follow up at end of treatment was $85 \%$.

\section{Intervention}

Families were allocated to enhanced $(\mathrm{n}=76)$, standard $(\mathrm{n}=77)$, and self directed BFI $(\mathrm{n}=75)$, or wait list control $(\mathrm{n}=77)$. Parents in the standard and enhanced BFI groups received, on average, 10 and 12 training sessions, respectively in 17 core child management strategies. Enhanced group parents also received training in partner or social support and coping skills. Parents in the self directed group were given training material on the management strategies and how to set and monitor behaviour change goals. Practitioners with advanced mental health training implemented the enhanced and standard interventions.

\section{Main outcome measures}

Maternal reported disruptive behaviour (change of $\geqslant 1.96$ standard errors of measurement from baseline in the Eyberg Childhood Behavior Inventory [ECBI] and the Parent Daily Report checklist [PDR]), clinician observed reduction in disruptive behaviour of $\geqslant 30 \%$, and change from clinical to normal range on the ECBI.

\section{Main results}

Maternal rating of clinically important changes in the ECBI were reported for more children in all the BFI groups than the control group (64\% for enhanced, $49 \%$ for standard, and $47 \%$ for self directed BFI $v 24 \%$ for control group, $\mathrm{p}<0.01$ for all comparisons with control). Maternal PDR showed that the enhanced and standard BFI groups had greater improvements in behaviour compared with the control group (43\% for enhanced, $25 \%$ for standard, 12\% for self directed BFI, and 3\% for controls). Only the enhanced BFI group had a higher rate of $\geqslant 30 \%$ reduction in child disruptive behaviour compared with the control group (57\% v 37\%; 48\% for standard BFI, $29 \%$ for self directed BFI). More children in the BFI groups changed designation from clinical to normal than did controls ( $71 \%$ for enhanced, $56 \%$ for standard, and $56 \%$ for self directed BFI $v 31 \%$ for controls).

\section{Conclusions}

Several formats of a behavioural family intervention (Triple P-Positive Parenting Program) reduced disruptive behaviours in 3 year old children at risk for developing conduct problems. The enhanced group reported the most consistent changes.

*See glossary.

\section{COMMENTARY}

This study by Sanders $e t$ al represents a sophisticated and progressive addition to parent based intervention studies aimed at high risk children who have disruptive behaviours. Of particular importance are the benefits of enhancing basic parenting programmes by addressing associated risks, the added effect of having a practitioner involved in structured parenting programmes, and the apparent dose response relation. The multiple outcome measures helped to show the robustness of the enhanced intervention. However, the mixed outcomes for the standard and self directed BFI weaken positive endorsement, particularly given the lack of correction for multiple statistical testing.

The policy implications of this study are complicated. It used targeted advertisements as the recruitment strategy. Although the intake assessment determined that the sample had clinically important levels of problems, this was not a clinically referred population. This is important because some evidence shows that those recruited from advertisements have better outcomes than patients who are referred from a clinic despite similar levels of psychopathology. ${ }^{1}$ Therefore it is unknown whether the more intense levels of the Triple-P programme would be sufficient for change in clinic based populations. At the other end, the wide dissemination of this programme may have some limits for prevention and early intervention purposes. The standard and enhanced programmes included home visitation and the use of highly trained mental health specialists, which could make it too costly for dissemination for large samples. It will be useful to know whether the Triple-P programme would have similar positive outcomes using clinicians with less advanced training. For example, a different study described the cost effectiveness of using early childhood educators or bachelor level trained personnel to provide a community based, group parent training programme. ${ }^{2}$

John D McLennan, MD, MPH Canadian Centre for Studies of Children at Risk McMaster University and the Hamilton Health Sciences Corporation Hamilton, Ontario, Canada

1 Brent DA, Kolko DJ, Birmaher B, et al. Predictors of treatment efficacy in a clinical trial of three psychosocial treatments for adolescent depression.J Am Acad Child Adolesc Psychiatry 1998;37:906-14.

2 Cunningham CE, Bremner R, Boyle M. Large group community-based parenting programs for families of preschoolers at risk for disruptive behaviour disorders: utilization, cost effectiveness, and outcome. J Child Psychol Psychiatry 1995;36:1141-59. 